\title{
Determination of Matrix Element Sodium in the Sodium Metavanadate by Inductively Coupled Plasma Atomic Emission Spectrometry
}

\author{
CHENG Yong \\ PanGang Group Research Institute Co., Ltd., State Key Laboratory of Vanadium and Titanium Resources \\ Comprehensive Utilization, \\ Panzhihua 617000, Sichuan, china \\ e-mail: luck2005_1@sina.com and chengyongpg@163.com
}

\begin{abstract}
The sodium metavanadate samples are digested in hydrochloric acid, and the content of matrix element sodium is directly measured by inductively coupled plasma atomic emission spectrometry (ICP-OES). The optimum dilution ratio is disposable obtained by optimizing the weight of the sample and the volume of the solution, so the sodium element detection signal intensity are regulated and controlled to a moderate level, in order to ensure that the method has good dynamic linear range. The paper have investigated the matrix effects, spectral interferences and background effects which caused by the coexistence of high concentrations of vanadium matrix, and then by taking preferred nebulizer pressure, plasma excitation power, observation height and other ICP spectrometer operating parameters, as well as optimizing and selecting the sodium element analysis spectral lines and its background correction and detection integration region, and combined with matrix matching and synchronization background correction measures, thus have effectively eliminated the transmission of the test solution injection, aerosol formation, ICP balance and excitation, background noise, element determination as well as many other interferences that caused by the alkali metal ions, high vanadium matrix and an argon molecule spectral band and other factors. The results show that: the calibration curve showed a good linear relationship when the sodium concentration below $20 \%$, the correlation coefficient is equal to 0.9975 ; RSD is less than $1.0 \%$; recovery rate is $95.5 \%$ to $103.5 \%$. The determination results of the actual sample by ICP-OES method are consistent with flame atomic absorption spectrometry.
\end{abstract}

Keywords-inductively coupled plasma atomic emission spectrometry (ICP-OES); sodium metavanadate; matrix element; sodium; constant analysis;

\section{INTRODUCTION}

Sodium metavanadate is an important basic chemical materials, it not only is the basic raw materials which used to manufacture ammonium vanadate, potassium metavanadate and other products, or is intermediate control products of producing of vanadium pentoxide, but also mainly is used as chemical reagents, catalysts, driers and dye mordant. The sodium content in sodium metavanadate is one of the key technical indicators for the evaluation of the quality of products, the current analysis method is reference to existing determination of the trace sodium or potassium in the vanadium pentoxide and ammonium metavanadate samples by flame atomic absorption spectrometric(F-AAS), have not seen reported in the literature that determination of sodium in sodium metavanadate by inductively coupled plasma atomic emission spectrometry (ICP-OES). This paper tests the analysis method that determination of matrix main element sodium in the sodium metavanadate by ICP-OES. The sodium metavanadate samples are digested in hydrochloric acid and the optimum dilution ratio is disposable obtained by optimizing the weight of the sample and the volume of the solution, and take no any intermediate dilution steps, therefore the sodium element detection signal intensity are regulated and controlled to a moderate level so that ensure that the method has good dynamic linear range, and simplify the operation to shorten the process, improve the analysis speed, precision and accuracy. The paper take preferred nebulizer pressure, plasma excitation power, observation height and other ICP spectrometer operating parameters, as well as optimizing and selecting the sodium element analysis spectral lines and its background correction and detection integration region, improve detection performance of the easily ionized and excited alkali metal sodium ion and reduce the influence of background noise, combined with matrix matching and the synchronous background correction measures in order to eliminate interference effects of the high vanadium matrix and argon molecule spectral band, without using the internal calibration of the complicated operation, the method is easy operation, less short process steps and has excellent technical performance indicators.

\section{EXPERIMENTAL}

\subsection{Instruments and reagents}

iCAP 6300 type the full spectrum of direct-reading plasma atomic emission spectrometer, Elix water purifier.

Superior grade purity hydrochloric acid; High purity vanadium pentoxide and sodium carbonate (purity $>99.99 \%$ ) and was prepared into a concentration $1 \mathrm{mg} / \mathrm{mL}$ single-element standard stock solution.

\subsection{Instrument conditions}

ICP spectrometer working conditions: RF power $750 \mathrm{~W}$, auxiliary gas flow rate $(\mathrm{Ar}) 1.0 \mathrm{~L} / \mathrm{min}$, nebulizer pressure of $0.26 \mathrm{MPa}$, peristaltic pump speed $60 \mathrm{r} / \mathrm{min}$, observation height $11.6 \mathrm{~mm}$, each measurement integrator 
3 times and each integration time 10s.

\subsection{Test Method}

Weighing $0.1000 \mathrm{~g}$ sodium metavanadate sample into a $250 \mathrm{~mL}$ Teflon beaker, first add into $10 \sim 15 \mathrm{~mL}$ water to disperse sample, and then add into $5.0 \mathrm{~mL}$ hydrochloric acid, and until the digestion reaction was complete at a low temperature heating conditions, and then boiled at a high temperature to produce a uniform large bubbles holding for 2 to 3 minutes. The test solution was cooled to room temperature and transferred to a $500 \mathrm{~mL}$ volumetric flask, was diluted with water to volume. Finally, was measured in the above instrument working conditions.

\section{RESULTS AND DISCUSSION}

\subsection{Spectral interference test}

Sodium element is easily ionized and excited and has fewer atomic emission line, mainly includes the $\mathrm{Na}$ $589.592 \mathrm{~nm}, \mathrm{Na} 330.237 \mathrm{~nm}, \mathrm{Na} 330.298 \mathrm{~nm}$ and $\mathrm{Na}$ $568.820 \mathrm{~nm}$, and the main sensitive spectrum is located in the visible light region. In order to eliminate the impact of the vanadium matrix and argon molecular bands, therefore through spectral interference test, the appropriate sodium analysis spectral and background correction locations and detection area are all selected.

The experiments show that: within sodium analysis line scan window of $\mathrm{Na} 589.592 \mathrm{~nm}$, the $0.08 \mathrm{mg} / \mathrm{mL}$ vanadium standard solution (about $\omega_{\mathrm{V}}=40 \%$ ) do not produce peak in the sodium analysis line detection integral or background correction area, its signal scanning line is a straight signal baseline, but the intensity is higher than the reagent blank. This shows that the coexistence matrix element vanadium does not produce spectral interference in determination of sodium, but the matrix effect and background noise and other influencing factors that produced by the high content of vanadium result in background baseline of the sodium analysis line is elevated and the signal intensity is increased, and thus have some interference on the determination, therefore, the synchronous background correction and matrix matching is used to eliminate the impact. As shown in Figure 1.

In addition, although the sensitivity of $\mathrm{Na}$ $330.237 \mathrm{~nm}$ and $\mathrm{Na} 330.298 \mathrm{~nm}$ are worse than $\mathrm{Na}$ $589.592 \mathrm{~nm}$, and the influence of vanadium matrix is greater, its still can be used as the spectral lines that determination of the high content of sodium. But $\mathrm{Na}$ $568.820 \mathrm{~nm}$ due to poor sensitivity and is subject to the vanadium matrix serious interference, it can not be used for the determination of sodium in sodium metavanadate.

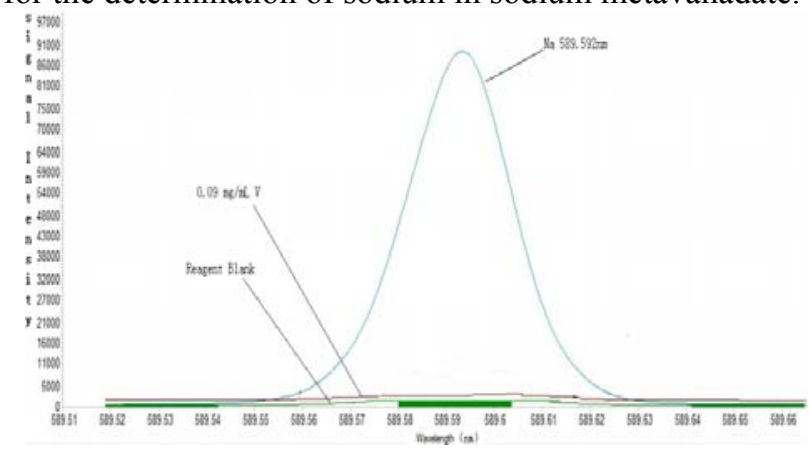

Figure 1. Na $589.592 \mathrm{~nm}$ interference test chart

\subsection{Analytical wavelength select}

According to the above tests, it is preferred that elemental analysis wavelength and background correction position, and the results are shown in TABLE 1.

TABLE1 THE ANALYSIS SPECTRAL LINES AND BACKGROUND CORRECTION AREA OF THE SODIUM（NM）

\begin{tabular}{llcc}
\hline Wavelength & $\begin{array}{c}\text { The left } \\
\text { background } \\
\text { correction area }\end{array}$ & $\begin{array}{c}\text { The left } \\
\text { background } \\
\text { correction area }\end{array}$ & $\begin{array}{c}\text { Detection } \\
\text { integration } \\
\text { region }\end{array}$ \\
\hline 589.592 & $589.520-589.540$ & $589.640-589.660$ & $589.580-589.600$ \\
330.237 & $330.196-330.206$ & $330.230-330.245$ & $330.230-330.240$ \\
\hline
\end{tabular}

\subsection{The calibration curve and its detection range test}

According to contain the sodium element 0.0, 5.0, $10.0,15.0,18.0,20.0 \%$ concentration relationship, a concentration gradient series sodium element calibration curve standard solutions were prepared by diluting the sodium element standard stock solution of $1 \mathrm{mg} / \mathrm{mL}$. Then, in accordance with the concentration from low to high in the order to be determined and the calibration curve was fitted to the drawing, see Fig 2.

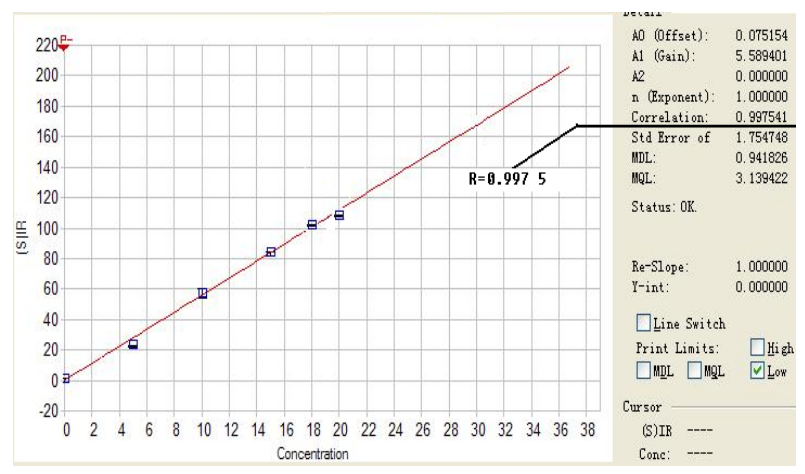

Figure 2. Na 589.592nm calibration curve

From Figure 2, in the sodium concentration of the element is $20 \%$ or less, the calibration curve showed a good linear relation, the correlation coefficient is equal to 0.9975 , so the method meet the needs of the determination matrix main element sodium in the sodium metavanadate.

\subsection{The precision test}

Choose two sodium metavanadate samples randomly, and each sample was eight separate digestion and determination in parallel experiments, mathematical statistics of the results to calculate the relative standard deviation, and the results are shown in TABLE 2.

TABLE 2 THE ACCURACY EXPERIMENTS RESULTS OF METHOD

\begin{tabular}{ccccccc}
\hline $\begin{array}{c}\text { Determin } \\
\text { ation of } \\
\text { the }\end{array}$ & \multicolumn{2}{c}{ Sample 1 } & & \multicolumn{2}{c}{ Sample 2 } \\
\cline { 2 - 3 } \cline { 5 - 6 } $\begin{array}{c}\text { number } \\
\text { of time }\end{array}$ & $\begin{array}{c}\mathbf{N 3 0 . 2 3 7} \\
\mathbf{n m}\end{array}$ & $\begin{array}{c}\mathbf{5 8 9 . 5 9 2} \\
\mathbf{~ n m}\end{array}$ & & $\begin{array}{c}\mathbf{3 3 0 . 3 7 2} \\
\mathbf{n m}\end{array}$ & $\begin{array}{c}\mathbf{N 8 9 . 5 9 2} \\
\mathbf{n m}\end{array}$ \\
\hline 1 & 19.02 & 18.94 & & 18.76 & 18.74 \\
2 & 19.02 & 19.04 & & 18.85 & 19.04 \\
3 & 19.10 & 19.10 & & 19.07 & 19.20 \\
4 & 19.10 & 19.01 & & 19.23 & 19.01 \\
\hline
\end{tabular}




\begin{tabular}{ccccc}
\hline 5 & 19.14 & 18.86 & 18.99 & 18.86 \\
6 & 19.02 & 18.80 & 18.72 & 18.80 \\
7 & 19.15 & 19.04 & 18.79 & 19.14 \\
8 & 19.14 & 18.75 & 18.84 & 18.75 \\
$\begin{array}{c}\text { Average } \\
(\%)\end{array}$ & 19.09 & 18.94 & 18.91 & 18.94 \\
$\begin{array}{c}\text { Standard } \\
\text { deviation } \\
\text { Relative } \\
\text { standard } \\
\text { deviation } \\
(\%)\end{array}$ & 0.058 & 0.127 & 0.176 & 0.179 \\
\hline
\end{tabular}

Seen from TABLE 2, the relative standard deviation (RSD) is less than $1.0 \%$ that indicates the method has good precision and good reproducibility measurement results. The measurement results of the Na330.237nm and Na589.592nm are basically consistent. If the final test result is the average of the two analytical lines, this can further improve the precision and accuracy of the detection data.

\subsection{The recovery of the method}

Two actual sodium metavanadate products (sample 3 and sample 4) are analyzed in accordance with the experimental methods and the spike recovery is tested, the results are shown in TABLE 3.

TABLE 3 RECOVERY TEST RESUlTS (\%)

\begin{tabular}{ccccc}
\hline Sample & $\begin{array}{c}\text { Found before } \\
\text { add standard }\end{array}$ & Added & $\begin{array}{c}\text { Found after a } \\
\text { dd standard }\end{array}$ & Recovery \\
\hline 3 & 19.44 & 2.00 & 21.35 & 95.5 \\
4 & 17.89 & 4.00 & 22.03 & 103.5 \\
\hline
\end{tabular}

From TABLE 3, we know that recovery of the tested element is between $95.5 \%$ and $103.5 \%$, which indicate that the results of the method are accurate and reliable, with good accuracy.

\subsection{Analysis of the results of controlled trials}

The actual sodium metavanadate products (sample 5 and sample 6) were detected by this method (ICP-OES), and then were compared with the flame atomic absorption spectrometry (F-AAS), in order to verify the accuracy of the method. The results are shown in TABLE 4.

TABLE 4

ANALYSIS RESULT COMPARISON

$(\%)$

\begin{tabular}{ccccc}
\hline \multirow{2}{*}{ Element } & \multicolumn{2}{c}{ Sample 5 } & \multicolumn{2}{c}{ Sample 6 } \\
\cline { 2 - 5 } & ICP-OES & F-AAS & ICP-OES & F-AAS \\
\hline $\mathrm{Na}$ & 14.80 & 14.63 & 11.98 & 12.20 \\
\hline
\end{tabular}

According to TABLE 4, the ICP-OES and F-AAS analysis results are basically the same and the deviation between two this methods is very little, this indicates that the method is accurate and reliable.

\section{CONCLUSIONS}

The matrix element sodium in the sodium metavanadate is determined by ICP-OES, this paper summary matrix effects, spectral interference, background noise and other influencing factors in high concentrations of vanadium coexistence system, through taking a series of preferred sample dilution ratio, appropriate analysis spectral lines and its detected integral and background correction region and other measures, the detection signal intensity of the high concentration sodium is regulate moderate, so that effectively expand the detection scope of the method and the accuracy and precision of this method have improved. Method has the advantages of simple operation and short inspection cycle, and reduces labor intensity and human resource consumption, and better meet requirements of the fast-paced production site for product quality inspection and control.

\section{REFERENCES}

[1] Ferrous Metallurgy Industry Standards of the People's Republic of China [S] YB/T 5335-2006. Vanadium Pentoxide-Determination of sodium oxide and potaiumss oxide content-The flame atomic absorption spectrometric method.

[2] Feng Zhongping. Determination of sodium sulfur iron phosphorus in the vanadium oxide by inductively coupled plasma atomic emission spectrometry [J]. Metallurgical analysis, 2010 Vol.30 (3):30-33.

[3] Anthemidis A N, Ioannou K I. Development of a sequential injection dispersive liquid-liquid micro extraction system for electrothermal atomic absorption spectrometry by suing a hydrophobic sorbent material: Determination of lead and cadmium in natural waters $[\mathrm{J}]$. Analytica Chimica Acta, 2010, 668(1):35-40.

[4] Liu GuoHong. Simultaneous determination of Al, Fe, Ca, As, Na $\mathrm{K}$, $\mathrm{Si}$ in the ammonium metavanadate by ICP-OES [J]. Western China Science and Technology, 2011.10 (22): 19-20.

[5] Yang X H, Wei J F, Liu H T, etal. Direct determination of trace elements in tungsten products using an inductively coupled plasma optical emission charge coupled device detector spectrometer [J]. Spectrochimica Acta: Part B, 1998, 53:1405-1412.

[6] Kostadinova E, Velichkov S, Daskalova N. Spectral interferences in the determination of traces of scandium, yttrium and rare earth elements in pure rare earth matrices by inductively coupled plasma atomic emission spectrometry: Part B, 2000, 55: 689-729.

[7] Ito K, Masukawa F, Mao Q L, et al. Inductively coupled plasma-atomic emission spectral fitting analysis of rare-earth elements in ion-adsorption type ores [J]. Anal. Chim. Acta, 1988, 362(2-3):241-245.

[8] BrownC P, Hilies C R. Determination of trace impurities in high zirconium by emission spectrograghy [J]. Can. J. Spectrosc.1975, 20(6):145-149.

[9] Aleksieva L, Daskalova N, Velichkov S. Spectral interferences in the determination of traces of scandium, yttrium and rare earth elements in pure rare earth matrices by inductively coupled plasma-atomic emission spectrometry: Part VI-Ytterbium [J]. Spectrochimica Acta: Part B, 2002, 57:1339-1350.

[10] Bahattin Gmgum, Gandan Hamamci. Determination of traces of some rare elements in phosphate rocks by inductively coupled plasma atomic emission spectrometry [J]. Spectrocopy Letters, 1991, 4(9):1229-1236 